\title{
Propuesta de la ISO 21001 en el entorno educativo de Aguascalientes, México
}

\section{Proposal of ISO 21001 in the educational environment of Aguascalientes, Mexico}

\author{
DURÓN-DE LUNA, Abelardo $\dagger$, AGUIRRE-TÉLLEZ, Wendy Miriam, ESTRADA-NAVARRETE, \\ Jorge Manuel y MUÑOZ-DÍAZ Ismael
}

Universidad Tecnológica de Aguascalientes, Blvrd Juan Pablo II, La Cantera, 20200 Ex hacienda, Aguascalientes Mex.

ID $1^{\text {er }}$ Autor: Abelardo, Durón-De Luna / ORC ID: 0000-0003-4584-2693

ID $1^{\text {er }}$ Coautor: Wendy Miriam, Aguirre-Téllez / ORC ID: 0000-0002-4057-8793

ID $2^{\text {do }}$ Coautor: Jorge Manuel, Estrada-Navarrete / ORC ID: 0000-0001-6641-9363

ID $3^{\text {er }}$ Coautor: Isamel, Muñoz-Díaz / ORC ID: 0000-0003-2779-8932

DOI: $10.35429 / J O T E .2019 .9 .3 .8 .16$

Recibido 17 de Julio, 2019; Aceptado 24 de Septiembre, 2019

\section{Resumen}

La norma ISO 21001:2018 (ISO, International Organization for Standarization) vista como Sistema de Gestión Normativo, creada para el sector educativo en sus niveles formativos (Básico, Media Superior y Superior) en la que suministran, comparten o transfieren, conocimientos, habilidades y destrezas para estudiantes mediante formación práctica, elemental, técnica y/o investigación y cuyos destinatarios de los productos y servicios de estas organizaciones son los estudiantes, así como las partes interesadas y clientes (Padres, administración, representantes de la sociedad, industria y otras organizaciones educativas con las que se establezcan acuerdos de colaboración) a las que estas entidades se comprometen a atender y satisfacer, en virtud de sus propios objetivos, basándose en 11 principios, los 7 ya conocidos de la ISO 9001:2015 y 4 más basados en "Responsabilidad Social", "Equidad y Responsabilidad", "Conducta ética" y "Seguridad y protección de datos"; todo ello contribuirá a la creación de una mejora continua, controlada y estratificada en las organizaciones Educativas.

\begin{abstract}
The ISO 21001: 2018 standard (ISO, International Organization for Standardization) seen as a Regulatory Management System (MS), created for the education sector at its training levels (Basic, Higher and Higher Media) in which they provide, share or transfer, knowledge, skills and skills for students through practical, elementary, technical and / or research training and whose recipients of the products and services of these organizations are students, as well as interested parties and clients (Parents, administration, representatives of society, industry and others educational organizations with which collaboration agreements are established) to which these entities undertake to meet and satisfy, based on their own objectives, based on 11 principles, the 7 already known of ISO 9001: 2015 and 4 more based on "Social Responsibility", "Equity and Responsibility", "Ethical Conduct" and "Security and data protection"; All this will contribute to the creation of a continuous, controlled and stratified improvement in Educational organizations (MSEO).
\end{abstract}

MSEO, ISO 21001:2018, Responsibility 


\section{Introducción}

ISO es una serie de directrices y estándares internacionales diseñados para ser utilizados para la evaluación y certificación de organizaciones que han implementado un sistema de calidad. (Página oficial ISO) Los estándares ISO se basan en el concepto de que ciertas características mínimas de un sistema de gestión de la calidad podrían ser útilmente estandarizadas. Esto brindará un beneficio mutuo a los proveedores y clientes, y el enfoque no se centra solo en la calidad del producto sino también en los procesos para lograr productos de calidad.

Se puede ver como un procedimiento de control de gestión que implica documentar los procesos, la producción y la distribución para garantizar que la calidad de los productos y servicios, satisfaga las necesidades de los clientes.

Otras características que el ISO proporciona, es el requisito para que la organización se concentre, para lograr objetivos de calidad auto determinados, para proporcionar servicios con una planificación adecuada y posteriormente para medir y controlar esta prestación de servicios (Camilleri, A. -2014-).

La norma ISO 21001:2018 cuenta con cuarenta y nueve definiciones básicas, dentro de las esenciales para este trabajo mencionamos las siguientes: organización que la define como la persona o a un grupo de personas que tiene sus propias funciones con responsabilidades, autoridades y relaciones para lograr sus objetivos; define a las partes interesadas como un grupo de interés ya sea a una persona o a una organización que puede verse afectada por una decisión o actividad; así mismo, define al sistema de gestión como un conjunto de elementos interrelacionados entre sí, de una organización para establecer políticas, objetivos y los respectivos procesos para lograr esos objetivos; define la efectividad como el punto de medición en el que se realizan las actividades planificadas y se alcanzan los resultados planificados y Define a los procesos como una interacción de actividades de entrada y salida (SIPOC), entre otras.
Durante el desarrollo del trabajo se mencionará al pensamiento lateral, este hace mención al éxito o fracaso de las personas que dentro de las organizaciones pesan no sólo el pensamiento, sino también las emociones, los sentimientos, las actitudes, los valores, las creencias, las percepciones, la inteligencia, la cultura, la creatividad, el conocimiento, la experiencia, entre otros aspectos. Este tipo de pensamiento lateral no se rige por las secuencias lineales que limitan la percepción y la acción, no ignora alternativas, opciones o aspectos que no encajen dentro del esquema. Por el contrario, regala la posibilidad de beneficiarse de estos, flexibilizando la percepción y la manera de obrar y actuar. En materia de aprendizaje el pensamiento lateral potencia los procesos que conducen a la apropiación, uso, generación y comprensión de conceptos y conocimientos (Arboleda, Julio César. 2007).

Dentro de la Norma ISO 21001:2018, se mencionan 11 Principios, los cuales entablan una relación de enfoques primarios estilizados en la formación de alto nivel educativo; esto es, que los procesos y en si los sistemas deben estar enfocados al estudiante $\mathrm{y}$ otros beneficiarios, con un liderazgo visionario, un compromiso con las personas involucrados en el sistema, con un enfoque a procesos, o sea, que el sistema a aplicar en la organización educativa deberá estar conformada por proceso (SIPOC), relacionada a la mejora continua y a decisiones basadas en evidencias; gestionando relaciones con el contexto organizacional, con responsabilidad social corporativa, accesibilidad y equidad, así como conducta ética y seguridad y protección de datos.

La norma ISO 21001:2018 evoca a un modelo de gestión sistemático referenciado a la calidad y mejora de servicio de las instituciones educativas, entendiendo por ellas los preescolares, escuelas primarias, secundarias, bachillerato, universidades, así como centros y/o departamentos de capacitación que con base a la norma ISO 9001:2015, proporciona un instrumento de servicio que atiende el mejoramiento de procesos, procedimientos $\mathrm{y}$ actividades, así como de servicios que las instituciones educativas proporcionan a los denominados clientes del sistema. 
$\mathrm{Su}$ estructura se basa en lo que se conoce en el mundo de la estandarización como estructura de alto nivel (HSL por sus siglas en Inglés), alineándola a sistemas de Gestión conocidos, como por ejemplo la ISO 9001:2015, ISO 14001:2015, ISO 45001:2018, etc.; ello comprende una serie de pasos enumerados del 1 al 10, en el que se especifican una serie de lineamientos a cumplir, así: 1. Objeto y campo de aplicación; 2. Referencias normativas; 3 . Términos y definiciones; 4. Contexto de la organización; 5. Liderazgo; 6. Planificación; 7. Apoyos; 8. Operación; 9. Evaluación del desempeño y 10. Mejora.

$\mathrm{Al}$ igual que se enumeran una serie de Anexos en el documento: Anexo A. Requisitos adicionales para organizaciones de educación infantil; Anexo B. Principios del sistema de gestión de organizaciones educativas (SGOE); Anexo C. Clasificación de las partes interesadas en las organizaciones educativas; Anexo D. Directrices para la comunicación con las partes interesadas; Anexo E. Procesos, medidas y herramientas en las organizaciones educativas; Anexo F. Ejemplo de mapeo a estándares regionales; Anexo G. Consideraciones de salud y seguridad para organizaciones educativas.

En el estado de Aguascalientes, México, el Organismo que maneja la educación es el Instituto de Educación de Aguascalientes (IEA), definido como un organismo descentralizado del Gobierno del Estado, con personalidad jurídica y patrimonio propios, con responsabilidad sobre todos los tipos, niveles y modalidades educativos que se impartan, ofrezcan o promuevan en la entidad (Página Oficial del Gobierno del Estado de Aguascalientes y Página Oficial Instituto de Educación del estado de Aguascalientes. http://www.iea.gob.mx).

\section{Metodología a desarrollar}

Según la (International Organization for Standarization -2018-) norma ISO 21001:2018, es específica en los requisitos para un sistema de gestión para organizaciones educativas cuando dicha organización:

- Necesita demostrar su capacidad para apoyar la adquisición y el desarrollo de competencias mediante la enseñanza, el aprendizaje o la investigación.
- Tiene como objetivo mejorar la satisfacción de los estudiantes, clientes, otros beneficiarios y el personal a través de la aplicación efectiva de su institución, incluidos los procesos para mejorar el sistema y la garantía de conformidad con los requisitos de los estudiantes y otros beneficiarios.

- Todos los requisitos de ISO 21001: 2018 son genéricos y están destinados a ser aplicables a cualquier organización que utiliza un plan de estudios para apoyar el desarrollo de competencias a través de la enseñanza, el aprendizaje o la investigación, independientemente del tipo, tamaño o método de entrega.

- Se puede aplicar a organizaciones educativas dentro de organizaciones más grandes cuyo negocio principal no sea la educación, como los departamentos de capacitación profesional.

- No se aplica a organizaciones que solo producen o fabrican productos educativos.

La implementación de la Norma ISO 21001:2018 en organismos educativos y centros de capacitación, plantea el homologar y sistematizar los servicios de calidad de manera eficiente, profesional y holística en pro de lograr la satisfacción de los clientes definidos por el sistema, gobierno y de la sociedad en general; buscando que el sector Educación y los servicios de capacitación y consultoría maduren; dando como ventajas aspectos como la internacionalidad, la competitividad, la transportabilidad, el diseño curricular, la continuidad de estudios, el intercambio de profesores y/o administrativos, entre otros. $\mathrm{La}$ norma ISO 21001:2018 es la primera norma específica para organismos de educación, anteriormente sistema y modelos como la ISO 9001:2015 (SGC), ISO 29990:2010 (Servicio de aprendizaje y formación no formal), IWA:2007 (Guía para la aplicación de la norma ISO 9001 en la educación), NTC 5555:2007 (SGC para instituciones de formación para el trabajo), GTC 200:2005 (Directrices para aplicar ISO 9001 establecimientos de educación básica), entre otras, se utilizaban para enmendar a las organizaciones educativas a contener algún sistema de gestión y en ocasiones controlar ciertos procesos y procedimientos dentro de su propio sistema. 
Igualmente, en México, las Normas CONOCER (Consejo Nacional de Normalización y Certificación de competencias laborales), es una entidad paraestatal sectorizada en la Secretaría de Educación Pública (SEP), con un órgano de gobierno tripartita con representantes de los trabajadores, los empresarios y el gobierno federal, por tiempo se ha dedicado a realizar normas para ser aplicadas a las organizaciones educativas como los siguientes estándares de competencia (EC): EC 0782 Aplicación de habilidades genéricas para la competitividad laboral; EC 0647 Propiciar el aprendizaje Significativo en Educación media superior, EC 0847 Promoción de servicios de educación inicial, EC 0646 Facilitación de procesos de aprendizaje bajo el enfoque por competencias en Educación media superior, EC 0604 Atención educativa para Adultos Mayores, EC 0730 Elaboración de la ruta de mejora para una institución educativa, EC 0150 Coordinación de procesos en Gestión educativa estratégica en Centros Escolares de Educación Básica, EC 0786 Evaluación del aprendizaje con enfoque formativo, EC 0477 Tutoría en la Educación Media Superior y Superior, EC 0992 Coordinación de los Subsistemas educativos en la formación Dual, EC0554 Trabajo en equipo, EC0507 Liderazgo para la autonomía de gestión, EC0489 Facilitación de procesos de innovación, EC0513 Aprender a aprender y EC0422 Administración de las personas en las organizaciones. Para la aplicabilidad de la norma ISO21001:2018 es necesario tener conocimientos de Mejora Continua (ISO 9001:2015, ISO 10002:2007, ISO 19011:2018), de Gestión de Procesos (Análisis de procesos de negocio -BPA-, Modelo y notación de Procesos de Negocio-BPMN-), entre otros; de Gestión de Riesgos (ISO 31000, ISO 31010, ISO 31100); Medición del cumplimiento de objetivos (Balance Score Card); Mejora del desempeño (ISO 9004); Excelencia en la Gestión (Modelos de excelencia en la gestión, Premio Nacional de Calidad, Premio Iberoamericano de la calidad, Malcom Baldrige, Entre otros); Planeación estratégica, ISO 37001 Sistemas de Gestión Anti Soborno, ISO 44001 SG de las relaciones colaborativas de las organizaciones, ISO 20400 Compras sostenibles, ISO 26000 Responsabilidad Social, ISO 56002 Directrices sobre el SG de la Innovación, ISO 56003 Herramientas y métodos para asociación de innovación, tomando únicamente lo que le concierne a la organización.
Al aplicar sistema de gestión en instituciones educativas, centros de enseñanza y de capacitación, un error es considerar al Alumno o persona a tomar capacitación como el cliente del sistema (Hernada, J. M. C., \& Gaya, C. G.), las escuelas tienen como objetivo fundamental entregar graduados a la sociedad (Cliente) y en referencia a los centros de capacitación, tendrán como objetivo el de entregar personas con adquisición de conocimientos, habilidades y destrezas a la empresa que contrato el servicio, por lo tanto, el Alumno y/o persona que toma algún curso, es el producto que entregan los proveedores al sistema de Gestión de la organización.

Para lograr una implementación significativa, es importante el aplicar un liderazgo con sentido común, que sea incluyente e involucre al personal para la realización de tareas, que reafirme un compromiso con la organización, así como el que asegure los recursos necesarios para su implementación; es menester comentar que el personal deberá contar con las competencias adecuadas a la parte del proceso involucrada, para cumplir con la subcláusula 7.2 de la norma ISO 21001:2018. Así como la de garantizar la eficiencia de su actividad dentro del sistema.

Es importante considerar que se debe realizar los procesos adecuados para evitar el que se engrose, ya que una organización con una gran cantidad de procesos se hace pesada para la evaluación del sistema.

La capacitación, haciendo recurrencia a la cláusula 7 "Soporte" de la norma ISO 21001:2018, específicamente a la subcláusula 7.2 Denominada "Competencias" deberá focalizarse a las necesidades del proceso, así como del mismo sistema, todo ello para cumplir con las exigencias de eficiencia del sistema, así como de las prerrogativas y exigencias de los clientes. 
Las consideraciones en base a la experiencia de un servidor en su Implantación de la Norma ISO 21001:2018 para cualquier organismo educativo del estado de Aguascalientes es a grosso modo la de Formar equipo de trabajo, Entrenamiento y Capacitación: "Análisis e interpretación de ISO 21001:2018"; incluye enfoque a procesos, liderazgo, planeación estratégica, PDCA, partes interesadas y riesgos; Análisis de brechas de Cumplimiento, marco estructural, cronograma de actividades; Determinación de Objetivos mediante metodologías específicas (SMART); Creación de Políticas y Alcance del programa; Generación de Indicadores para medir efectividad, eficiencia y eficacia del sistema; Capacitación en desarrollo de procesos, procedimientos y técnicas y habilidades de especificaciones de trabajo y Capacitación en Auditoría Interna ISO 19011: 2018 y Project Plan (ISO 21001).

Así mismo, la propuesta va enmarcada en la utilización de las siguientes Metodologías y estrategias para implementar ISO 21001: 2018:

1.- Planeación estratégica: también llamada alineación estratégica. A través de este proceso, se definen la visión, misión, metas y objetivos a lograr por la organización, para marcar el camino a seguir por la misma.

2.- Honshi Kanri: esta es una metodología japonesa, que lo que busca es realizar el despliegue de la estrategia. Esta herramienta consiste en definir el rumbo estratégico de la organización, desplegarlo en forma de cascada por los diferentes niveles y regresar para poder tener retroalimentación para poder efectuar los pertinentes ajustes. De esta manera logramos involucrar todos los niveles.

3.-Análisis PESAT: es un análisis de la situación Política, Económica, Social, Ambiental y Tecnológica. Esta herramienta nos permite clasificar desde una perspectiva de temas de interés, el propio análisis.
4.- Análisis FODA: bien conocido por todas las organizaciones, esta metodología se centra en hacer un reconocimiento a nivel tanto interno, mediante la búsqueda de las fortalezas y debilidades de la organización, como a nivel externo, tratando de adelantarse a posibles amenazas y oportunidades. De aquí, se derivan objetivos y estrategias. Esto permite dar cumplimiento al punto 4.1 de la norma ISO 9001:2015, referido a la comprensión del contexto de la organización, además se recomienda el método CAME (Corregir, Afrontar, Mantener y Afrontar) para dar respuesta al FODA.

5.- Matriz MEFE (Matriz de Evaluación de Factores Externos): esta herramienta de evaluación es algo más detallada, dándole al análisis un carácter más específico a la vez que cuantitativo.

6.- Matriz MEFI (Matriz de Evaluación de Factores Internos): al igual que la anterior, esta metodología es más detallada a la hora de analizar los factores internos y también aporta un carácter cuantitativo.

7.- Análisis de los Stakeholders: mediante esta herramienta podemos identificar quiénes son los stakeholders o partes interesadas de nuestra organización y permite determinar el nivel de interés que tienen cada uno de ellos, así como la influencia que pueden tener sobre nuestra organización. Por ejemplo, en algunas organizaciones, sobretodo en algunas pertenecientes al Sector Público, los sindicatos gremiales podrían ser una de estas partes interesadas o stakeholders muy importante. En otras organizaciones, un stakeholders podría ser un cliente relevante, cuya decisión o influencia puede ser vital para la organización. En definitiva, con esta herramienta podemos identificar estas partes interesadas y, en base a ello, poder definir las estrategias necesarias para su atención y poder tomar las decisiones de acuerdo a la situación existente.

8.- Definición del alcance: de acuerdo a los requisitos de la norma ISO 9001:2015, el alcance de la organización debe quedar documentado, bien sea en el propio manual de calidad o en aquel documento que estime la organización. 
9.- Mapeo y documentación de procesos: sin procesos definidos de manera adecuada, queda carta libre a la arbitrariedad. Por ello, a través del mapeo de procesos se identifican todos los procesos de la organización y sus interrelaciones. Es la base para la determinación de la documentación del SGC, permitiendo el seguimiento del mismo y la posibilidad de mejora continua.

10.- Interrelación de procesos: a través de esta herramienta, se busca analizar los lazos de unión existentes entre los diferentes procesos que se llevan a cabo en la organización.

\section{Caso Aguascalientes}

En Aguascalientes, en aspectos de Educación Básica (1), existe un porcentaje de analfabetismo del $1.1 \%$, encontrándonos en el lugar número 2 con respecto a los estados de la República Mexicana con menor valor; el porcentaje de Alumnos por género oscila en un $49.3 \%$ Femenino y un $50.7 \%$ Masculino, con un promedio de 12,578 Docentes en servicio y 5,090 Personal Auxiliar Administrativo, sin embargo, los males observados en el magisterio local, los maestros se sienten mal preparados, en el ciclo 2017-2018 desertaron el 10\% de los Alumnos de Secundaria y Preparatoria; El director del Instituto de Educación de Aguascalientes (IEA), Raúl Silva Perezchica, admitió que la deserción en los niveles de secundaria y media superior, siguen siendo uno de los puntos débiles de la enseñanza en el estado siendo la falta de motivación, mal desempeño pedagógico de los docentes y la economía, los principales factores. El director del IEA reconoció que en deserción escolar hay un área importante de oportunidad que debe ser atendida sobre todo en secundaria y educación media, mientras que en primaria y preescolar no parece haber mayores problemas.

En nivel Preescolar se cuenta con 663 Escuelas, con 2,368 Aulas, 2,251 grupos y 53,794 Alumnos en el ciclo escolar 2018-2019; se incluyen las escuelas comunitarias rurales de CONAFE. En el mismo ciclo, para Primaria, el Estado de Aguascalientes cuenta con 706 escuelas, 5814 Aulas, 5,081 grupos, con 158,832 Alumnos y Para el nivel Secundaria, se cuenta con 368 escuelas, 2,935 Aulas, 2,537 grupos y 73,117 Alumnos.
La implementación de un SGOE en base a la norma ISO 21001:2018 para las organizaciones en Aguascalientes estaría comprendidas a groso modo de procesos como los siguientes: 'Revisión de la Dirección' (cl.5); Metodología 'Acciones para abordar riesgos y oportunidades' (cl. 6.1); 'Control de personal' (cl.7.1.2; 7.2); 'Soporte técnico' (cl.7.1.3.b); 'Control de monitoreo y medición de recursos' (cl.7.1.5); 'Comunicación interna' (cl.7.3); 'Control de información documentada' (cl.7.5.); 'Análisis del contrato' (cl.8.2); 'Comunicación con el cliente' (cl.8.2.1); 'Diseño y desarrollo de productos y servicios' (cl.8.3); 'Control de proveedores externos de productos y servicios' (cl.8.4); "Producción y prestación de servicios" (cl.8.5. y 8.6); 'Control de productos y servicios de procesos no conformes' (cl.8.7); 'Auditoría interna' (cl.9.2) y 'Acción correctiva' (cl 10.2); donde cl significa clausula.

En visto a los párrafos anteriores, es menester comentar que cada uno de los problemas mencionados pudiera controlarse a través de la aplicación de esta Normatividad, como por ejemplo: la deserción; esto es, ya implementado el SGOE, uno de los procesos obligatorios es el referente a la cláusula 6.1 que es un procedimiento para determinar los riesgos del sistema, en el deberá observarse la o las metodologías con las que el sistema evidenciara en base a la ISO 31010, acto seguido se aplicaría la metodología en cuestión y se evaluaría con los requerimientos de la clausulas 'Control de productos y servicios de procesos no conformes' (8.7) y 'Acción correctiva' (10.2), si es un acto recurrente se evaluaría en mejora continua (10.3). Esto pudiera llegar a cambiar en base a los procesos y procedimientos implantados en la organización, ya que al menos se considera que cada subsistema tendría planes y programa diferentes, mas sin embargo ciertos procedimientos pudieran ser similares como por ejemplo en los Sistemas Conalep con respecto al sistema del Colegio de Estudios Científicos y Tecnológicos del Estado de Aguascalientes (Cecytea) ambos de Bachillerato, pudieran tener alcances (cl 4.3) similares, quizás la misma Política de Calidad (cl 5.2), los mismos objetivos (cl 6.2), quizás las mismas competencias genéricas (cl 7.2), su misma prestación de productos y servicios (cl 8.5 ), liberación de productos y servicios ( $\mathrm{cl}$ 8.6), etc. 
Esto dependerá entre otras cosas de la similitud dentro del contexto de la organización, virilizando el enfoque a que unos de los clientes sea el Gobierno del estado de Aguascalientes, otro sería la sociedad de Aguascalientes, sintetizando, se realizaría un Plan de Mitigación a las estrategias definidas por la organización que tratan de reducir la probabilidad de ocurrencia del riesgo o reducir el impacto que pueda causar. Es importante entender que el objetivo de mitigación de riesgos es reducir la exposición al riesgo con la intención de llevarlo a los límites de los umbrales aceptables para cada organización.

Para ello, es importante hacer mención que la aplicación del SGOE no resulta una varita magita mágica en la resolución de problemas cuando regularmente ocurren fenómenos como los siguientes:

Designación de un responsable sin la autoridad debida, Procedimientos mal explicados, formatos inadecuados para registrar la información necesaria, Diseño de procesos engorrosos, Falta de dedicación y tiempo por parte del personal con conocimientos y responsabilidad para desarrollar adecuadamente el soporte documental del sistema, Falta de coherencia entre lo que se dice y lo que se hace, Falta de un buen sistema de medición organizativa o indicadores, Imposición Resistencia al cambio, entre otros factores.

\section{Caso Universidad Tecnológica vía coordinación Nacional de Universidades Tecnológicas (UUTT)}

Respecto a la Norma ISO 21001:2018, en su aspecto holístico e implementada, podría apoyar a la coordinación Nacional de Universidades Tecnológicas y Politécnicas (CGUTyP) a resolver el aspecto de investigación, aplicando una sistematización a un proceso homologado en la dicha coordinación para ser aplicado en cada una las universidades inscritas, esto es para estandarizar la forma de lograr investigaciones más adecuadas al termino tecnológico; en el Gráfico 2 se puede observar el status que presenta la Coordinación Nacional respecto al número de investigadores clase SNI por entidad federativa dentro de la República Mexicana, en el que se observa, que para el estado de Aguascalientes, en la actualidad no se cuenta con investigadores de este nivel, el caso en las universidades tecnológicas es complicado ya que se cuenta con los niveles de TSU e Ingeniería (Licenciatura) asesorar a estudiantes a nivel Maestría y ser Doctores y en las universidades Politécnicas si impartes nivel Maestría según se observa en el Gráfico 2.

La Norma ISO 21001:2018, especifica que la educación a distancia, e-learning o investigación serán incluidos como anexos normativos; haciendo hincapié en el tema de la investigación, esto es para fortaleces a la par, pero no inmiscuir para no tergiversar con los procesos de enseñanza/aprendizaje (PEA) que es el tema principal de esta norma; Así las métricas (KPI`s) estarán focalizada a fortalecer la calidad, desempeño y eficiencia de los productos a entregar en el proceso de Investigación; de esta manera tendríamos un estándar regulado por la coordinación para medir de manera clara, concreta y precisa a todo aquel investigador de cada nivel educativo o bien, por cada institución dentro de la misma coordinación. 


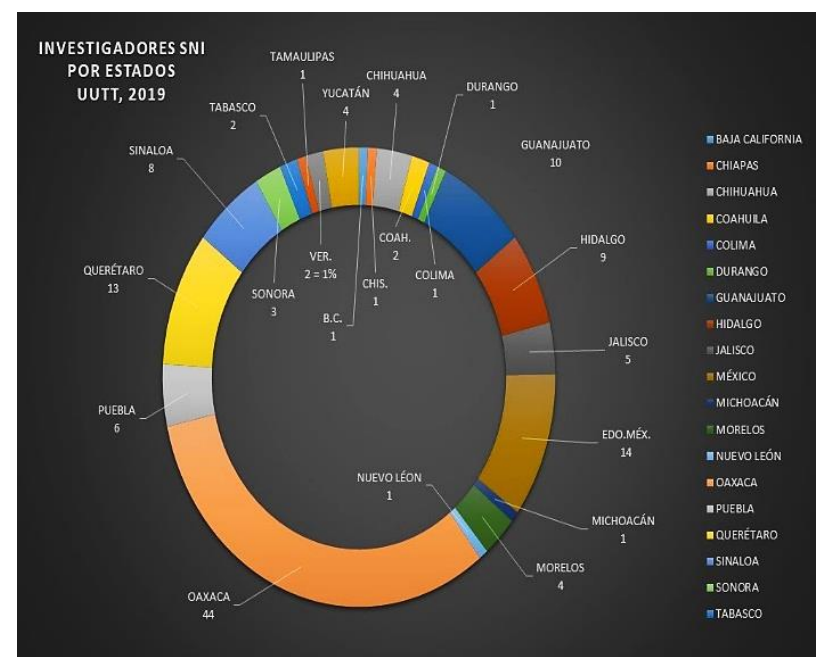

Gráfico 1 Investigadores SIN por estados UUTT 201

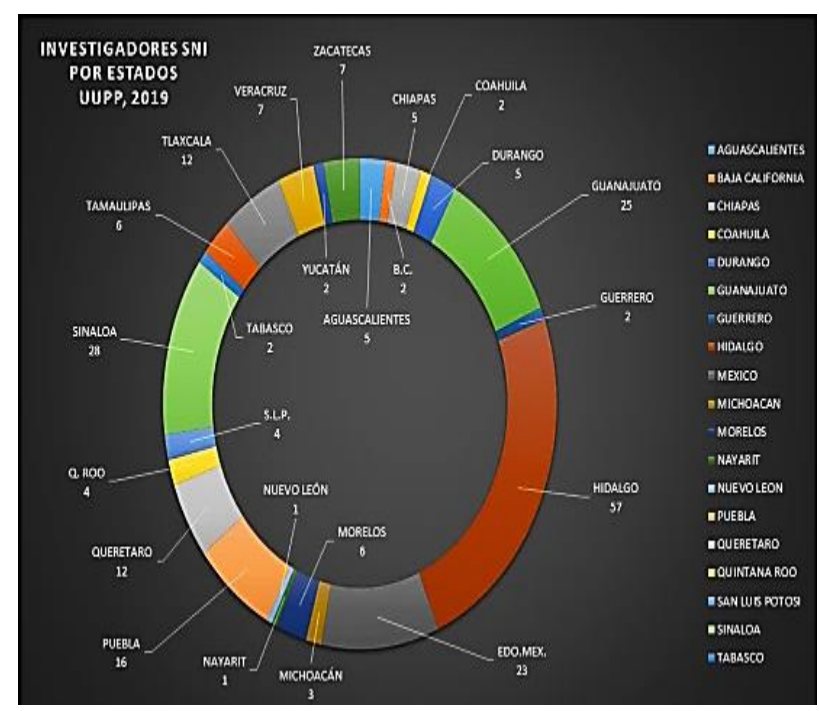

Gráfico 2 Investigadores SIN por estados UUPP 2019

\section{Conclusiones}

La Normativa de la familia ISO establece la forma para evaluar la capacidad de las organizaciones en rubros como diseñar, producir y entregar productos/servicios de calidad de manera eficiente; a través de métodos y actividades utilizados para asegurar que el cliente de la calidad de los productos y servicios que se le brindan, cumple con las especificaciones prometidas por el organismo, que en este caso es una institución académica o centro de capacitación.
En

\section{Aguascalientes}

existen organizaciones educativas dependientes del IEA, citare dos de ellas, la Comisión estatal de educación media y la Comisión estatal de educación superior, tienen por objeto coordinar las planeación y evaluación de los servicios que ofrecen el conjunto de las instituciones de educación que operan en el estado de Aguascalientes; entre otras tareas, autoriza lo que se conoce como el RVOE (Registro de un plan y programas de estudio ante la autoridad educativa, ya sea federal o estatal), que es una autorización para que el plan de estudios pueda ser impartido por una institución educativa; se hace mención a ello porque podría ser un medio para apoya a las instrucciones de educación media en el estado para implementar la normatividad ISO 21001:2018, previo haber tomado los cursos adecuados y las certificaciones necesarias para tal fin.

Este sistema de gestión para organizaciones educativas, facilitará la conducción racional de la organización educativa y permitirá velar por su eficacia en su desempeño. Los procesos de enseñanzaaprendizaje y el diseño y desarrollo curricular, serán sujetos a requerimientos de gestión específicos. Las ventajas para el personal capacitado en la ISO 21001:2018 será la de desarrollar habilidades y destrezas para: Diseñar, implantar y gestionar todo lo que comprenda este sistema de calidad; Gestionar proyectos de implantación que tengan relación con el estándar en las organizaciones, Aplicar técnicas para el manejo de los principios con la finalidad de mejorar continuamente una base educativa.

La implementación de esta norma traería ventajas competitivas a las empresas, al gobierno y a la sociedad en general, ya que al homologar los procesos, por ejemplo en las escuelas primarias, ya sean particulares o públicas, se supone que al ingresar al nivel secundaria, los Alumnos deberían tener los conocimientos mínimos necesarios para el siguiente nivel, se hablaría del formatos similares en cada plantel, etc. 


\section{Referencias}

Arboleda Aparicio, Julio César (2007). Pensamiento lateral y aprendizaje

Camilleri, A. (2014). Standardising Management Systems for Educational Organizations - implications of ISO 21001 for European Higher Education. Recuperado de https://eua.eu/component/attachments/attach ments.html?task=attachment\&id=1077 [ Links ]

\section{CONOCER.}

https://conocer.gob.mx/acciones_programas/co nocer-mision-vision-politica-objetivos-calidad/

Hernada, J. M. C., \& Gaya, C. G. (2013).

ISO 10002:2014(E) Quality Management Customer Satisfaction - Guidelines for complaints handling in organizations International Organization for Standardization. (2018).

ISO 21001:2018 - Educational organizations -Management systems for educational organizations -- Requirements with guidance for use. Ginebra: ISO. [ Links ]

ISO 26000, Guidance on Social Responsibility. British Standards Institution. (2015). Major revision to ISO 9001. Metal Powder Report, 70(5), 218. [ Links ]

ISO 9001, Quality management systems Requirements

ISO 9001:2015 (2015). Sistemas de gestión de la calidad. Estados Unidos Mexicanos.

Las cifras de la educación. Estadísticas de educación básica 2019-2019. Gobierno del estado de Aguascalientes.

Página Oficial Instituto de Educación del estado de Aguascalientes._http://www.iea.gob.mx

Página Oficial ISO. https://www.iso.org/home.html

UNESCO, 2011, International Standard Classification for Education 